$236 \longrightarrow$ LA VENTANA, NÚM. $48 / 2018$

\title{
BOTÁNICA: UNA CIENCIA FEMENINA EN LATINOAMÉRICA
}

TATIANA LOBATO DE MAGALHÃES ${ }^{1}$

\footnotetext{
${ }^{1}$ Universidad Autónoma de Querétaro,

México. Correo electrónico:

tatilobato@gmail.com
}

\section{Resumen}

La Botánica fue considerada la primera área de la Ciencia adecuada para las capacidades cognitivas de las mujeres. En el siglo XIX, mientras los hombres participaban en las expediciones naturalistas, las mujeres estaban limitadas a clasificar y herborizar el material colectado. Sin embargo, sus nombres no eran citados en los trabajos científicos, y de esta manera, un gran número de mujeres tuvieron "carreras invisibles". Por otro lado, la Botánica fue también una oportunidad para la participación de las mujeres en el mundo científico. La contribución femenina en la Botánica difiere de los otros ramos de la Ciencia. El objetivo fue analizar el papel actual de la mujer botánica en Latinoamérica. Fueron analizados datos de 1,567 artículos científicos, 448 herbarios y 10 sociedades botánicas en 19 países. A pesar del consenso que la Botánica es una "actividad intrínsecamente femenina", se observó que la participación femenina como curadoras de herbarios, presidentas de sociedades y congresos botánicos, así como autoras en publicaciones científicas, es equivalen- 


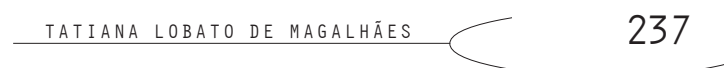

te a la participación masculina. La participación no está balanceada igualmente entre regiones. Argentina, Brasil y México presentaron mayor número de herbarios y publicaciones científicas, así como mayor participación de las mujeres, comparado con otros países latinoamericanos.

Palabras clave: carreras invisibles, ciencia igualitaria, género y Ciencia, publicación botánica.

\begin{abstract}
Botany was considered the first scientific area suited to women's cognitive understanding. In the $19^{\text {th }}$ century, while men took part in naturalistic expeditions, women's participation was limited to the analysis and classification of the plants and materials that were collected. Women's names were not mentioned in scientific works, and as such, a great number of women had "invisible careers". The scientific contribution to botany by women differs from their contributions to other branches of science. This article analyzes the present-day role of women botanists in Latin America using data gathered from 1,567 scientific articles, 448 herbariums, and 10 botanical societies from 19 Latin American countries. Although the consensus is that Botany is an inherently women-dominated branch of science, this article demonstrates that female participation as curators of herbaria, presidents of botanical societies and conferences, as well as authorship in scientific
\end{abstract}




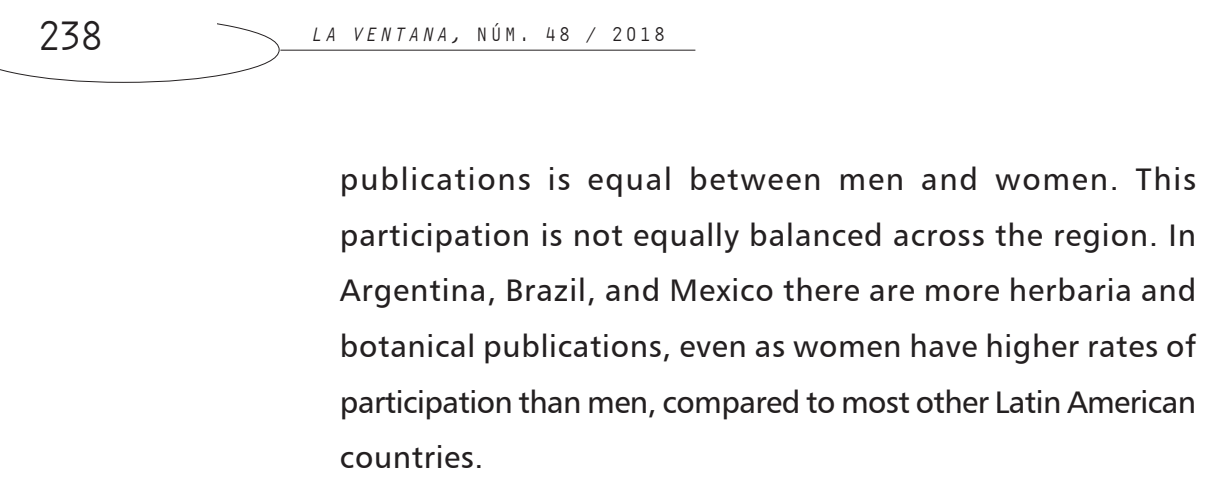

Keywords: invisible careers, equal science, gender and science, botanical publications.

RECEPCIÓN: 20 DE MARZO DE 2017 /ACEPTACIÓN: 19 DE JUNIO DE 2017

\section{INTRODUCCIÓN}

El género en la Ciencia es un asunto poco explorado en los estudios antropológicos. La primera vez que se escribió acerca de género y Ciencia fue en 1985 (Keller, 1985). En Latinoamérica hay registro de solamente 15 artículos publicados y tres libros acerca de este tema (Minella, 2013). La visión de género de la sociedad interfiere en la propia construcción de la Ciencia (Silva, 1998). Hasta el siglo XVIII, la visión predominante de la sociedad occidental fue que la Botánica se presentaba como la única Ciencia adecuada para la capacidad cognitiva femenina (Shteir, 1997; Blachford, 2013). Fue frecuente que las mujeres recibieran una educación informal y su aporte a la Botánica no fue reconocido públicamente (Shteir, 
1999). En esa época, la Botánica fue asociada a la figura femenina por John Miller con una representación de Flora, la diosa de las flores (Miller, 2010). Eso fue uno de los primeros registros de la intersección entre género, artes y Ciencia (Shteir, 2007). La participación de la mujer en la Botánica fue evidente desde su nacimiento, sin embargo, esta participación disminuyó con la profesionalización de esta Ciencia (Shteir, 1999; Sedeño, 2000).

En el siglo xIx hubo un mayor reconocimiento de las mujeres en la Ciencia, así como la aceptación de la primera latinoamericana María Augusta Generoso Estrella como estudiante en una universidad, en 1881 (Palermo, 2006). Desde entonces la participación femenina en la Ciencia ha crecido. La práctica de las ciencias naturales fue abierta al igualitarismo tanto de género, como de edad y origen social (Vega, 2014). En el siglo XIx la Botánica fue considerada como una actividad femenina (Shteir, 1997), una Ciencia de amateurs y hasta como un entretenimiento de la aristocracia (Leite, 2000; Blachford, 2013, Vega, 2014). La Botánica representaba una perspectiva religiosa-moral del estudio de la naturaleza (Catsikis, 2009). Tal vez por eso hubo un cierto direccionamiento de la Botánica hacia las mujeres, pues se presentaba como una actividad de carácter pasional, un tipo de distracción con bajos objetivos científicos. La mujer ha sido continuamente disminuida en la historia de la Ciencia (Tosi, 1998), ya que fue vista como ser intelectualmente inferior, que representaba una dependencia 
económica y psicológica del género masculino (Wollstonecraft, 2009).

En Latinoamérica, aún son pocos los estudios acerca de la historia de las mujeres naturalistas viajeras (Minella, 2013). Hay relatos de que algunas mujeres viajaron en América Latina, describiendo la naturaleza en sus bitácoras de viaje y por medio de pinturas e ilustraciones. Estas viajeras Ilevaban una bolsa con cartas de recomendación para ser aceptadas y muchas veces dormían a la intemperie (Leite, 2000). Las grandes expediciones naturalistas fueron hechas por hombres, mientras que las mujeres se limitaron a herborizar y clasificar las plantas colectadas en dichos viajes (Lopes, Sousa y Sombrio, 2004). En el siglo xIX en las Américas hubo relatos de la existencia de 1,185 mujeres con algún tipo de interés en la Botánica, sin embargo, solamente el $15 \%$ tuvieron algún reconocimiento profesional (Rudolph, 1982). A los hombres que enfrentaron la dureza del campo en beneficio de la Ciencia se les concedió el atributo de héroe, sin embargo, a las mujeres cuando máximo fueron reconocidas como aventureras (Lopes, 1998). Se considera que la participación femenina en las expediciones fue mayor de lo que se ha registrado (Sombrio, Lopes y Velho, 2008), una vez que un gran número de mujeres naturalistas tuvieron carreras invisibles en la Ciencia (Lopes et al., 2004).

La contribución científica que las mujeres aportaron a la Botánica difiere de su papel en otras áreas de la Ciencia. La 
publicación de estudios básicos de Botánica hechos por mujeres permitió la inserción femenina a la Ciencia y su reconocimiento de autoría científica (George y Martín, 2011). Actualmente las mujeres son quienes más publican en Botánica (Bredenkamp y Smith, 2008). Por otro lado, los hombres dominan la producción científica, en general ellos son quien más publican y son los más citados (Sugimoto, Larivière, Gingras, y Cronin, 2013). En algunos países como Brasil las mujeres dominan en número entre los investigadores de la Botánica (Guedes, Azevedo y Ferreira, 2015). Hoy y siempre la influencia femenina en el manejo de la biodiversidad contribuye a la conservación de los recursos genéticos vegetales, sobre todo de las especies de uso económico y alimenticio (Howard, 2003). Las mujeres se especializan más en algunas áreas de la Botánica, como los estudios etnobotánicos, las plantas medicinales y la ilustración botánica (Leite, 2000).

Los objetivos de la investigación fueron analizar la actual participación femenina en la Botánica en países de Latinoamérica (Argentina, Bolivia, Brasil, Chile, Colombia, Costa Rica, Cuba, Ecuador, El Salvador, Guatemala, Honduras, México, Nicaragua, Panamá, Paraguay, Perú, República Dominicana, Uruguay y Venezuela) y retratar la cuestión de género en esa área de la Ciencia. Fueron consultados trabajos con relación a género y Ciencia en Latinoamérica, género y Botánica, premios concedidos a mujeres botánicas, así como los siguientes datos: a) registro de herbarios Latinoamericanos en la base de datos 
internacional en 2017 (NYBG Steere Herbarium, n.d.) y su relación de género entre los curadores; b) sociedades botánicas Latinoamericanas nacionales; c) relación de género entre presidentes de las sociedades Botánicas en 2017; d) género de los presidentes de congresos nacionales de Botánica en su última edición; e) género en publicaciones de revistas científicas de las sociedades botánicas nacionales desde 2010 a 2016; f) registro de revistas de botánica de países Latinoamericanos en la base de datos Latindex (Sistema Regional de Información en Línea para Revistas Científicas de América Latina, el Caribe, España y Portugal, 1997). Los datos encontrados fueron compilados en cuadros y gráficas.

\section{MUJERES BOTÁNICAS EN}

\section{LATINOAMÉRICA}

Latinoamérica está definida como el futuro "continente femenino", presentando una proporción femenina de progresivo aumento (Alves, 2014) y las mujeres han representado un importante papel en el desarrollo de la Botánica. Marianne North fue considerada una viajera naturalista pionera, quien exploró Brasil a finales del siglo XIX. Ella relató su experiencia naturalista en bitácoras de viaje y pintó una serie de cuadros sobre las flores, las plantas y la vegetación en general. Marianne North colectó también ejemplares botánicos para el herbario Kew Garden de Londres, sin embargo, eso está citado de manera informal en la literatura. Su trabajo, sobre 
todo sus escritos, han sido prácticamente ignorados, son de los pocos relatos de viaje de una mujer naturalista en Latinoamérica en esa época. Dickenson (2015) considera que Marianne North fue la precursora de la actual preocupación del ambiente natural y la conservación de la biodiversidad. Asimismo, entre los siglos $x x$ y $x x ı$ hubo mujeres que se destacaron como pioneras en la Botánica Latinoamericana en Argentina, Brasil, Costa Rica, Guatemala, México y Panamá (Cuadro 1).

Cuadro 1. Ejemplos de mujeres que se destacaron en la Botánica Latinoamericana

\begin{tabular}{|c|c|c|c|}
\hline Nombre & País & Período & Contribuciones principales \\
\hline Nélida María Bacigalupo & Argentina & 1924 -actual & Taxonomista \\
\hline Bertha Lutz & Brasil & $1894-1976$ & $\begin{array}{c}\text { Grande incentivadora na inserción da mujer } \\
\text { a la Ciencia en Brasil }\end{array}$ \\
\hline Graziela Maciel Barroso & Brasil & $1912-2003$ & Taxonomista, profesora e investigadora \\
\hline Veridiana Victoria Rossetti & Brasil & $1910-2010$ & $\begin{array}{c}\text { Primera agrónoma brasileña e importante } \\
\text { científica }\end{array}$ \\
\hline Adelaida Chaverri Polini & Costa Rica & $1947-2003$ & $\begin{array}{l}\text { Investigación y conservación de los bosques } \\
\text { de altitud y de los Páramos }\end{array}$ \\
\hline Elfriede de Poll & Guatemala & 1922 - actual & Pesquisa etnobotánica y enseñanza \\
\hline Helia Bravo Hollis & México & $1901-2001$ & $\begin{array}{l}\text { Especialista en cactáceas y considerada una } \\
\text { de las más importantes Botánicas del siglo xx } \\
\text { en México }\end{array}$ \\
\hline Luz María Villarreal de Puga & México & $1913-2013$ & $\begin{array}{l}\text { Pionera en la conservación de los recursos } \\
\text { naturales mexicanos }\end{array}$ \\
\hline $\begin{array}{l}\text { Magdalena Josefina Peña } \\
\text { Muñoz }\end{array}$ & México & $1938-2012$ & $\begin{array}{l}\text { Primera presidenta mujer de la Asociación } \\
\text { Botánica Mexicana, incentivadora de la } \\
\text { creación de sociedades botánicas }\end{array}$ \\
\hline Mireya Correa & Panamá & 1940 - actual & $\begin{array}{c}\text { Taxonomista e investigadora, fundadora del } \\
\text { herbario de la Universidad de Panamá }\end{array}$ \\
\hline
\end{tabular}


Nélida María Bacigalupo fue una botánica de Argentina vinculada al Instituto de Botánica Darwinian. Ella describió muchas especies botánicas y en 2007 recibió el premio Mujer Ejemplar De San Isidro 2007 (Portal Uno Argentina, n.d.), como un reconocimiento a su legado en la Botánica argentina.

En Brasil se destacaron Berta Lutz, Graziela Maciel Barroso y Veridiana Victória Rossetti. Bertha Lutz fue la precursora de la inserción de las mujeres a la Ciencia en Brasil, además de su significativa contribución para la conservación del medio ambiente y de la biodiversidad (Sombrio et al., 2008). Graziela Maciel Barroso fue considerada la primera dama de la Botánica, una de las más importantes botánicas de Brasil y fue la primera mujer a ser seleccionada como naturalista en el Jardín Botánico de Río de Janeiro. A pesar de iniciar su carrera a los 30 años de edad (continuó su labor hasta los 91 años), fue muy exitosa en su profesión y se le reconoció con el premio Millenium Botany Award, conferido en el Congreso Internacional de Botánica (sBB Sociedade Botânica Do Brasil, 2012). Veridiana Victória Rossetti, formada como ingeniera agrónoma, trabajó aspectos de fitosanidad y citricultura, y fue reconocida internacionalmente como una de las mayores investigadoras en citricultura (Agência FAPESP, 2010).

En Costa Rica, Adelaida Chaverri Polini destacó por su aporte en la conservación de la biodiversidad de los bosques de montaña. Fue considerada una mujer destacada en Costa Rica (Camacho y Chavarría, 2007). En Cuba, Maritza García ganó el premio 
Julián Acuña 2016, de la Sociedad Cubana de Botánica, por su significativa contribución a la biología de la conservación y por el aporte al sistema nacional de áreas protegidas (Peláez, 2016). En Guatemala se destaca la botánica austríacaguatemalteca, Elfriede de Poll, directora del herbario de la Universidad del Valle de Guatemala, contribuyó en los estudios etnobotánicos en Guatemala y repetidas veces recibió homenajes nacionales (Calderón, 2016).

En México sobresalió Helia Bravo Hollis, especialista en las cactáceas, sin duda una de las figuras que más contribuyó en el desarrollo de la Botánica en este país. Fue también la primera mujer mexicana licenciada en Biología (Espinosa y Vargas, 2002). Luz María Villarreal de Puga fue considerada una botánica importante en el reconocimiento de la flora de México. Colectó más de 17,000 ejemplares botánicos y describió nuevas especies de maíz silvestre (Zamudio, 2014). También en México se destaca Magdalena Josefina Peña Muñoz por su papel en la defensa y divulgación de la biodiversidad mexicana. Fue la primera mujer presidenta de la Sociedad Botánica Mexicana, presidenta de la Asociación Mexicana de los Jardines Botánicos, apoyó el desarrollo de las Artes, Ciencia y Tecnología en México, una gran incentivadora de las asociaciones y la creadora del Calendario del Jardín Botánico. Durante décadas esta publicación proporcionó un espacio para que los artistas pudiesen representar su visión del reino vegetal y fue el precursor del calendario del Instituto de Biológica de la 
Universidad Nacional Autónoma de México, UNAM (Linares, 2012).

En el siglo XIX, en México varones crearon documentos impresos con contenidos de Zoología y Botánica para el público femenino, a citar: Calendario de las Señoritas Mejicanas (1839), Semanario de las Señoritas Mejicanas, Educación Científica, Moral y Literaria del Bello Sexo (1840-1842), Panorama de las Señoritas, Periódico Pintoresco, Científico y Literario (1842), Presente Amistoso (1847 y 1851), La Semana de las Señoritas Mejicanas (1851) y el Álbum de las Señoritas (1856) (Vega, 2014), demostrando el direccionamiento de la Botánica a las mujeres de esta época.

En Panamá recientemente se destaca Mireya Correa como ganadora del premio José Cuatrecasas de la Excelencia en Botánica Tropical 2008. Esta científica dedicó toda su vida a la Botánica. Fue docente, autora de libros, fundadora y directora del Herbario de la Universidad de Panamá (Smithsonian National Museum of Natural History, n.d.).

Muchas de las mujeres botánicas fueron personas que trabajaron hasta una edad avanzada, lo cual puede ser debido a que las mujeres necesitan más tiempo para su desarrollo profesional. Suele ocurrir que las mujeres sólo pueden dedicarse $100 \%$ a la actividad científica en una edad más avanzada, pues en su juventud comúnmente comparten sus actividades científicas con la maternidad. 


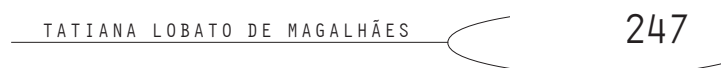

\section{CURADORAS DE HERBARIOS}

En Argentina, Bolivia, Brasil, Chile, Colombia, Cos-

ta Rica, Cuba, Ecuador, El Salvador, Guatemala, Honduras, México, Nicaragua, Panamá, Paraguay, Perú, República Dominicana, Uruguay y Venezuela se registran 448 herbarios en el listado internacional (NYBG Steere Herbarium, n.d.). Los países con mayor número de herbarios fueron Brasil (159 en el Index Herbariorum y 200 en la Rede Brasileira de Herbários, $\mathrm{RBH}$, según Vieira y Gasper, 2015), seguido por México (68), Argentina (53), Colombia (31) y Cuba (21). Entre los curadores listados están 219 mujeres (44\%) y 275 hombres (56\%). Solamente en Brasil, Ecuador, Guatemala y Panamá el número de mujeres curadoras superó a los hombres. Sin embargo, en Colombia, México y Perú el número de hombres listados como curadores fue mayor que el de mujeres. En los demás países los valores variaron al $50 \%$, demostrando un equilibrio de género (Figura 1). 


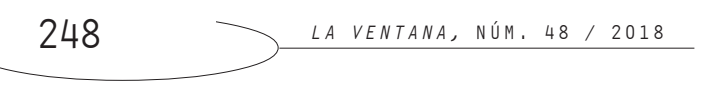

Figura 1. Relación de género de curadores de los herbarios de los países mencionados

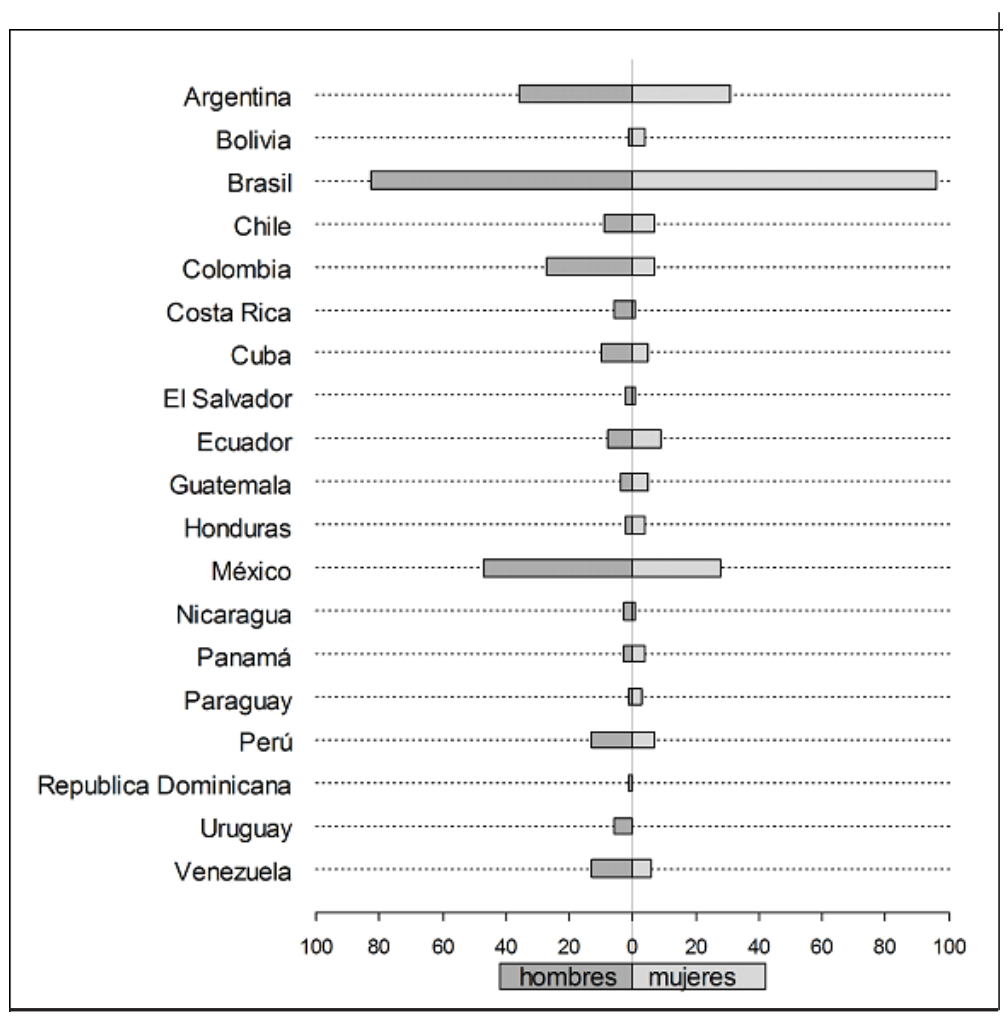

Fuente: adaptado de NYBG (2017), disponible en http://sciweb.nybg.org/

Science2/IndexHerbariorum.asp 


\section{MUJERES PRESIDENTES DE SOCIEDADES BOTÁNICAS Y ORGANIZADORAS DE CONGRESOS CIENTÍFICOS}

La Asociación Latinoamericana de Botánica fue fundada en 1978 con el objetivo de "responder a la imperiosa necesidad de mejorar la comunicación y las relaciones de trabajo entre los botánicos Latinoamericanos" (Asociación Latinoamericana de Botánica, 2017). Desde 1990 hasta los días actuales la Asociación Latinoamericana de Botánica fue presidida por cinco mujeres (71\%) y dos hombres (29\%), demostrando una mayor participación femenina (Cuadro 2).

Cuadro 2. Género de los presidentes de la Sociedad Botánica Latinoamericana

\begin{tabular}{|ccc|}
\hline Período & Paísde origen & Género del presidente \\
\hline $2015-2018$ & Ecuador & Mujer \\
$2010-2014$ & Brasil & Mujer \\
$2006-2010$ & Chile & Hombre \\
$2002-2006$ & Honduras-Repúblic aDominicana & Mujer \\
$1998-2002$ & Colombia & Hombre \\
$1994-1998$ & México & Mujer \\
$1900-1994$ & Chile & Mujer \\
\hline
\end{tabular}

Fuente: Adaptado de la Asociación Latinoamericana de Botánica (2017). 


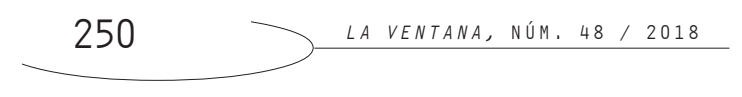

En relación con las sociedades nacionales de Botánica, entre los 19 países latinoamericanos considerados en este artículo, solamente 10 de estos presentaron sociedades organizadas: Argentina, Bolivia, Brasil, Chile, Colombia, Cuba, México, Paraguay, Perú y Venezuela. Actualmente estas sociedades son presididas por cinco mujeres y cinco hombres (Cuadro 3). Las sociedades botánicas de Brasil, Chile, Colombia, Paraguay y Perú son actualmente presididas por mujeres.

Cuadro 3. Género de los presidentes de las sociedades nacionales de Botánica en 2017

\begin{tabular}{|c|c|c|c|c|}
\hline País & Año de creación & $\begin{array}{c}\text { Género del presidente } \\
\text { en } 2017\end{array}$ & Revista científica de la Sociedad & Sitio en línea \\
\hline Argentina & 1945 & Hombre & $\begin{array}{l}\text { Boletín de la Sociedad Botánica Argentina } \\
\qquad \text { ISSN 1851-2372 }\end{array}$ & $\begin{array}{c}\text { http://www.botanicargentina } \\
\text {.com.ar/ }\end{array}$ \\
\hline Bolivia & 1994 & Hombre & $\begin{array}{l}\text { Revista de la Sociedad Boliviana de Botánica ISSN } \\
\qquad 2076-3190\end{array}$ & http://boliviabotanica.org/ \\
\hline Brasil & 1950 & Mujer & $\begin{array}{l}\text { Acta Botanica Brasilica } \\
\text { ISSN 0102-3306 }\end{array}$ & http://www.botanica.org.br/ \\
\hline Chile & 1977 & Mujer & $\begin{array}{l}\text { Gayana Botánica } \\
\text { ISSN 0717-6643 }\end{array}$ & $\begin{array}{c}\text { http://www2.udec.cl/ potani } \\
\text { ca/ }\end{array}$ \\
\hline Colombia & 1999 & Mujer & - & $\begin{array}{l}\text { http://www.asociacioncolom } \\
\text { bianadebotanica.org/ }\end{array}$ \\
\hline Cuba & - & Hombre & Revista del Jardín Botánico Nacional & - \\
\hline México & 1941 & Hombre & $\begin{array}{l}\text { Botanical Sciences } \\
\text { ISSN 2007-4476 }\end{array}$ & http://www.socbot.mx/ \\
\hline Paraguay & - & Mujer & - & - \\
\hline Perú & 1948 & Mujer & - & http://www.spebot.org/ \\
\hline Venezuela & 1971 & Hombre & $\begin{array}{l}\text { Acta Botánica Venezuelica } \\
\text { ISSN 0084-5906 }\end{array}$ & http://www.sbotanica.org.ve/ \\
\hline
\end{tabular}


En México, la Sociedad Botánica Mexicana fue creada en el año de 1941 y solamente tres mujeres ocuparon el cargo de presidente de la Sociedad. Ellas fueron: Magdalena Josefina Peña Muñoz (1976 a 1977), Patricia Dávila (1999 a 2001) y Victoria Sosa (2002 a 2004). En otras palabras, de los 75 años de la Sociedad Botánica Mexicana solamente 8 años fueron presididos por mujeres. Por otro lado, en Argentina, desde su creación en 1945, la Sociedad Botánica Argentina ha sido presidida por hombres. En Brasil, la Sociedad Botánica fue fundada en 1950 (Editorial, 2010), sin embargo, la participación femenina como presidentes de la Sociedad empezó en 1983 y fue bastante notoria a partir de la década de los ochentas.

El Congreso Latinoamericano de Botánica se presenta con frecuencia cuadrienal, el evento más reciente se llevó a cabo en el año de 2014, organizado por Brasil y fue presidido por la Dra. Tania dos Santos Silva (Asociación Latinoamericana de Botánica, 2017). Entre los 19 países analizados, solamente 10 presentaron eventos nacionales específicos de Botánica. Estos congresos nacionales de Botánica ocurren con diferente frecuencia: en Brasil y Chile son anuales, en Argentina y Colombia son bianuales, y en Bolivia, Ecuador, El Salvador, México, Perú y Venezuela, son trienales. En su última edición, los Congresos Nacionales de Botánica fueron presididos de forma equilibrada en relación a género (Cuadro 4). Brasil demostró mayor tradición en los congresos de Botánica, en su 
66 edición, seguido de Argentina (35 ${ }^{\text {a }}$ edición), Chile (26 edición), Venezuela ( $21^{\text {a }}$ edición) y México ( $20^{\text {a }}$ edición).

Cuadro 4. Género de los presidentes de Congresos nacionales de Botánica en su última ocurrencia

\begin{tabular}{|c|c|c|c|}
\hline País & Idioma & $\begin{array}{l}\text { Frecuencia/ edición más } \\
\text { reciente }\end{array}$ & $\begin{array}{c}\text { Género del presidente del más } \\
\text { reciente congreso }\end{array}$ \\
\hline Argentina & Español & Bianual / Xxxv & Hombre \\
\hline Bolivia & Español & Trienal / III & Hombre \\
\hline Brasil & Portugués & Anual / LxVI & Mujer \\
\hline Chile & Español & Anual / XxvI & Mujer \\
\hline Colombia & Español & Bianual / VIII & Hombre \\
\hline Costa Rica & Español & - & - \\
\hline Cuba & Español & - & - \\
\hline Ecuador & Español & $-/ v$ & Hombre \\
\hline El Salvador & Español & $-/ 1$ & ? \\
\hline Guatemala & Español & - & - \\
\hline Honduras & Español & - & - \\
\hline México & Español & Trienal / xx & Mujer \\
\hline Nicaragua & Español & - & - \\
\hline Panamá & Español & - & - \\
\hline Paraguay & Español & - & - \\
\hline Perú & Español & Trienal / xV & Mujer \\
\hline República Dominicana & Español & - & - \\
\hline Uruguay & Español & - & - \\
\hline Venezuela & Español & Trienal / XXI & Mujer \\
\hline
\end{tabular}




\title{
AUTORAS DE ARTÍCULOS
}

\begin{abstract}
CIENTÍ́FICOS
Las revistas científicas vinculadas a las Sociedades botánicas de los países Latinoamericanos fueron seleccionadas siempre y cuando cumplieran las siguientes características: ser indexada, arbitrada por pares, con periodicidad continua, con reconocimiento nacional/internacional y con artículos disponibles en los años más recientes. Fueron analizados un total de 1,567 artículos en cuanto a género de los autores (primer autor y colaboradores), publicados en los años de 2010 a 2016. Las revistas científicas consideradas en este artículo fueron: Boletín de la Sociedad Argentina de Botánica (20102016), Argentina; Sociedad Boliviana de Botánica (2010-2016), Bolivia; Acta Botanica Brasilica (2010-2016), Brasil; Gayana Botánica (2010-2016), Chile; Revista del Jardín Botánico Nacional (20102016), Cuba; y Botanical Sciences. Formerly Boletín de la Sociedad Botánica (2010-2016), México.

La relación de género se mostró proporcional entre la primera autoría de los artículos: 797 (51\%) hombres y 770 (49\%) mujeres. Por otro lado, entre los colaboradores se observó una mayor participación de varones, 2166 (56\%) hombres y 1694 (44\%) mujeres. En Argentina y Brasil, las mujeres fueron las que más publicaron como primeras autoras (Cuadro 5). En la búsqueda de artículos científicos específicos de Botánica de los países Latinoamericanos fueron encontradas 25 revistas (Sistema Regional de Información en Línea para Re-
\end{abstract}


Cuadro 5. Género entre autores y colaboradores de artículos entre los años de 2010 a 2016 en los periódicos de las Sociedades Botánicas de países Latinoamericanos

\begin{tabular}{|c|c|c|c|c|c|c|c|c|c|c|c|c|}
\hline \multirow[t]{3}{*}{ Revista } & \multicolumn{4}{|c|}{$\begin{array}{l}\text { Boletín de la Sociedad Argentina de } \\
\text { Botánica }\end{array}$} & \multicolumn{4}{|c|}{$\begin{array}{l}\text { Revista de la Sociedad Boliviana de } \\
\text { Botánica }\end{array}$} & \multicolumn{4}{|c|}{ Acta Botanica Brasilica } \\
\hline & \multicolumn{2}{|c|}{ Autor } & \multicolumn{2}{|c|}{ Colaborador } & \multicolumn{2}{|c|}{ Autor } & \multicolumn{2}{|c|}{ Colaborador } & \multicolumn{2}{|c|}{ Autor } & \multicolumn{2}{|c|}{ Colaborador } \\
\hline & mujer & hombre & mujer & hombre & mujer & hombre & mujer & hombre & mujer & hombre & mujer & hombre \\
\hline 2010 & 13 & 20 & 32 & 15 & -- & -- & -- & -- & 68 & 43 & 116 & 143 \\
\hline 2011 & 23 & 9 & 29 & 36 & 4 & 4 & 3 & 12 & 54 & 49 & 132 & 109 \\
\hline 2012 & 24 & 12 & 31 & 29 & 2 & 2 & -- & 3 & 53 & 50 & 129 & 149 \\
\hline 2013 & 5 & 9 & 61 & 50 & 1 & 3 & 1 & 5 & 44 & 44 & 117 & 123 \\
\hline 2014 & 32 & 28 & 64 & 38 & -- & -- & -- & -- & 40 & 32 & 75 & 101 \\
\hline 2015 & 29 & 22 & 79 & 38 & 5 & 3 & 5 & 2 & 33 & 35 & 77 & 93 \\
\hline 2016 & 31 & 28 & 83 & 71 & -- & -- & -- & -- & 39 & 35 & 84 & 107 \\
\hline Total & 157 & 128 & 379 & 277 & 12 & 12 & 9 & 22 & 331 & 288 & 730 & 825 \\
\hline Revista & \multicolumn{4}{|c|}{ Gayana Botánica } & \multicolumn{4}{|c|}{ Revista del Jardín Botánico Nacional } & \multicolumn{4}{|c|}{ Botanical Sciences } \\
\hline \multirow[b]{2}{*}{ Año } & \multicolumn{2}{|c|}{ Autor } & \multicolumn{2}{|c|}{ Colaborador } & \multicolumn{2}{|c|}{ Autor } & \multicolumn{2}{|c|}{ Colaborador } & \multicolumn{2}{|c|}{ Autor } & \multicolumn{2}{|c|}{ Colaborador } \\
\hline & mujer & hombre & mujer & hombre & mujer & hombre & mujer & hombre & mujer & hombre & mujer & hombre \\
\hline 2010 & 7 & 21 & 23 & 36 & -- & -- & -- & -- & 13 & 8 & 18 & 29 \\
\hline 2011 & 12 & 29 & 29 & 64 & 16 & 16 & 19 & 19 & 10 & 12 & 18 & 22 \\
\hline 2012 & 19 & 26 & 39 & 70 & -- & -- & -- & -- & 13 & 23 & 25 & 50 \\
\hline 2013 & 12 & 25 & 39 & 64 & 10 & 9 & 13 & 12 & 21 & 24 & 46 & 58 \\
\hline 2014 & 13 & 24 & 31 & 57 & -- & -- & -- & -- & 21 & 18 & 44 & 75 \\
\hline 2015 & 17 & 23 & 50 & 73 & 9 & 17 & 17 & 18 & 29 & 42 & 64 & 160 \\
\hline 2016 & 21 & 21 & 53 & 112 & -- & -- & -- & -- & 27 & 31 & 48 & 123 \\
\hline Total & 101 & 169 & 264 & 476 & 35 & 42 & 49 & 49 & 134 & 158 & 263 & 517 \\
\hline
\end{tabular}




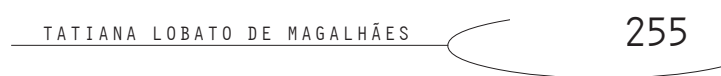

vistas Científicas de América Latina, el Caribe, España y Portugal, 1997). Entre los editores se registraron 19 hombres (76\%) y seis mujeres (24\%). México se destacó como el único país con equidad de género entre los editores (Figura 2).

Figura 2. Género de los editores de revistas de Botánica indexadas

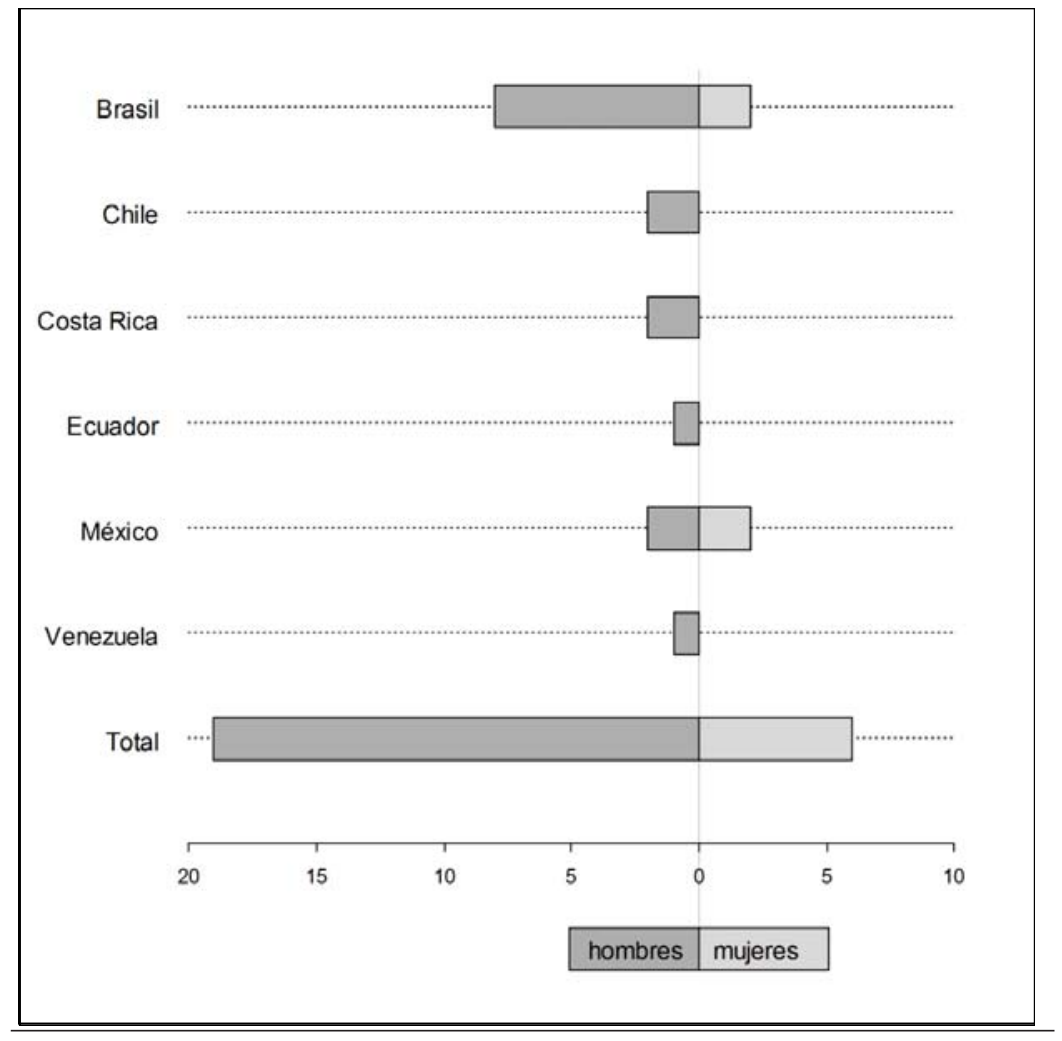

Fuente: Adaptado de Latindex (2017) 
CONSIDERACIONES FINALES

La Botánica ha sido considerada como Ciencia desde hace tres siglos. A pesar de esto muchos países Latinoamericanos no presentan Sociedades Botánicas, Congresos Botánicos o aún presentan un bajo número de herbarios y publicaciones científicas específicas de Botánica. Sin embargo, países latinoamericanos como Argentina, Brasil y México destacan por su número de herbarios y su organización de Sociedades y Congresos de Botánica.

En Brasil, en las pasadas décadas, las mujeres han participado más que los hombres en el Congreso Brasileño de Botánica (Pilon y Durigan, 2011), además, desde el 2001 las mujeres son mayoría (60\%) entre los becarios en Botánica del Consejo Nacional de Desarrollo Científico y Tecnológico de Brasil (Guedes et al., 2015). Pilon y Durigan (2011) sugieren que la predominancia de mujeres en la Botánica brasileña está asociada a la baja valoración de esta profesión en el país. Las mujeres se profesionalizan en las carreras con más bajo estatus o en carreras etiquetadas como femeninas, como es la Botánica (Velho y Léon, 1998).

El desarrollo femenino en una carrera científica presenta una tendencia inversamente proporcional al prestigio de esta actividad (Sedeño, 2000), ya que la Ciencia es considerada una "creación masculina" (Silva, 1998). Bredenkamp y Smith (2008) indican que actualmente las mujeres son las que más publican en Botánica, sin embrago con los datos que se ana- 


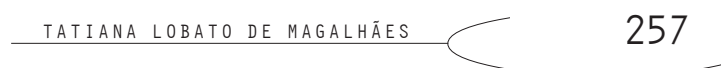

lizan en este escrito no necesariamente se observa esto. De manera general, Velho y Léon (1998) observaron que existen más mujeres actuando en el área de Biología, sin embargo, ellas son minoría en los cargos de mayor estatus, así como en las publicaciones científicas. Desde el punto de vista cualitativo, las mujeres tienen un aporte importante a la Botánica y desde el punto de vista cuantitativo, la participación femenina es equivalente al $50 \%$ para los cargos de liderazgo como curaduría, presidencia, autoría científica, lo que contradice que la Botánica sea una "Ciencia femenina". Sin embargo, es probable que aún en la actualidad existan mujeres con "carreras invisibles".

En Latinoamérica existe una disparidad de género en las publicaciones científicas, dónde los hombres se destacan como quienes más publican (Sugimoto et al., 2013). Sin embargo, el interés en la discusión de género en la Ciencia en Latinoamérica es un asunto reciente en la sociedad (Monteiro, 1997; Lopes et al., 2004). La participación de mujeres científicas asumiendo un papel protagonista en la Botánica de sus países es fundamental para el fortalecimiento, desarrollo y divulgación de la Botánica, asimismo como para el ascenso de las mujeres en la Ciencia en Latinoamérica. 
Bibliografía

Acta Botanica Brasilica. (2010-2016). Acta Botánica Brasilica. Belo Horizonte, Brazil. Recuperado de http://www.scielo.br/scielo.php? pid=01023306\&script $=$ sci_issues

AGÊNCIA FAPESP. (28 de diciembre de 2010). Victória Rossetti morre aos 93 anos. Recuperado de http://agencia.fapesp.br/ victoria_rossetti_morre_aos_93_anos/13243/

Alves, J. E. D. (5 de marzo de 2014). As desigualdades de gênero na América Latina e Caribe.

Ecodebate. Recuperado de https://www.ecodebate.com.br/2014/03/05/ as-desigualdades-de-genero-na-america-latina-e-caribe-artigo-dejose-eustaquio-diniz-alves/

Asociación LATINOAMERICANA de BotánICA. (2017). Junta directiva. Recuperado de http://www. botanica-alb.org/directiva.php

BLACHFORD, B. (2013). Exploring the history of women in botany: tracing seven female contributors of the UBC Herbarium. University of British Columbia. GEOC 429. Vancouver: University of British Columbia Library. doi: $10.14288 / 1.0075696$

BredenKamP, C. L. y SMIth, G. F. (2008). Perspectives on botanical research publications in South Africa: an assessment of five local journals from 1988 to 2002, a period of transition and transformation. South African Journal of Science, 11(104), 473-478.

CALderón, L. (2016). Entrevista a la Dra. De Pöll. Interamerican Network of Academies of Sciences, IANAS. Recuperado de http://www.ianas.org/ capacity_building/i_poll_spa.html 
Camacho de la O, A. L. y Chavarría, G. V. (2007). Mujeres destacadas de Costa Rica. San José, Costa Rica: Instituto Nacional de las Mujeres. Recuperado de http://www.inamu.go.cr/ documents/10179/11401/ Mujeres+destacadas + 2007.pdf/9ea 1 bbeb-3460-4e89-9e22$826 \mathrm{db} 1 \mathrm{ae} 88 \mathrm{c} 6 /$

CATSIKIS, P. J. (2009). A brilliant burst of botanical imagination: Proserpina and the nineteenth-century evolution of myth (Tesis doctoral). Faculty of Arts, University of Glasgow. Recuperado de http://theses.gla.ac.uk/ $774 /$

DiCKENSON, J. (2015). Marianne North: uma naturalista do século dezenove no Brasil? Cadernos Pagu, (15), 145-164.

EDITORIAL. (2010). Os sessenta anos da Sociedade Botânica do Brasil 19502010. Acta Botanica Brasilica, 4(24), 882.

Espinosa, P. y VARgAS, A. (2002). Helia Bravo, pioneira e inolvidable maestra. Biodiversitas, (40), 1-3.

Gayana Botánica. (2010-2016). Gayana Botanica. Chile: Facultad de Ciencias Naturales y oceanográficas, Universidad de Concepción. Recuperado de http://www.scielo.cl/scielo.php?script=sci_serial\&pid= 0717-6643\&lng =es\&nrm =iso

GEORGE, S. y MARTín, A. (2011). Botanising women: transmission, translation and European exchange. Journal of Literature and Science, 1(4), 1-11.

Guedes, M. de C., Azevedo, N. y Ferreira, L. O. (2015). A produtividade científica tem sexo? Um estudo sobre bolsistas de produtividade do CNPq. Cadernos Pagu, (45), 367-399. 
HoWARD, P. L. (2003). Women and the Plant World: an exploration. Women \& Plants: gender relations in biodiversity management \& conservation. Zed Books: London and New York.

Keller, E. F. (1985). Reflections on gender and science. New Haven: Yale University Press.

LeITE, M. L. M. (2000). Mulheres viajantes no século XIX. Cadernos Pagu, (5), 129-143.

LinARes, E. (2012). Magdalena Josefina Peña Muñoz. Botanical Sciences, 4(90), 481-483.

LOPES, M. M. (1998). Aventureiras nas ciências: refletindo sobre gênero e história das ciências naturais no Brasil. Cadernos Pagu, (10), 345368.

LOPES, M. M., SOUSA, L. G. P. y SOMBRIO, M. M. de O. (2004). A construção da invisibilidade das mulheres nas ciências: a exemplaridade de Bertha Maria Júlia Lutz (1894-1976). Revista gênero, 1(5), 97-109.

MinelLA, L. S. (2013). Temáticas prioritárias no campo de gênero e ciências no Brasil: raça/etnia, uma lacuna? Cadernos Pagu, (40), 95-140.

Miller, J. (2010). An Illustration of the Sexual System of Linnaeus. Estados Unidos: Kessinger publishing.

Monteiro, R. H. (1997). Inventing women science, technology and gender. Cadernos Pagu, (10), 445-448.

NYBG SteERE HeRBARIUM. (2017). Recuperado de http://sweetgum.nybg.org/ science/

Palermo, A. I. (2006). El acceso de las mujeres a la educación universitaria. Revista argentina de sociología, 7(4), 11-46.

Peláez, O. (26 de febrero de 2016). Confieren premio de la Sociedad 


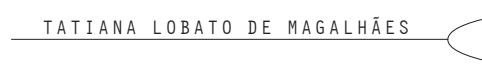

Cubana de Botánica. Granma. Recuperado de http://www.granma.cu/ ciencia/2016-02-26/confieren-premio-de-la-sociedad-cubana-debotanica-26-02-2016-23-02-10

PILON, N. A. L. y Durigan, G. (2011). A mulher na botânica: questão de gênero na participação feminina em congressos de botânica no Brasil. Hoehnea, 1(38), 115-121.

Portal Uno Argentina. (2007). Nélida María Bacigalupo: mujer ejemplar de San Isidro. Recuperado de http://www.portalunoargentina.com.ar/ noticiasver. asp? $\mathrm{id}=6449$

Revista Del Jardín Botánico Nacional. (2010-2016). Revista del Jardín Botánico Nacional. Recuperado de http://www.rjbn.uh.cu/index.php/RJBN

RUDOLPH, E. D. (1982). Women in nineteenth century american botany: a generally unrecognized constituency. American Journal of Botany, 8(69), 1346-1355. Recuperado de http://www.jstor.org/stable/ 2442761 ? seq=1\#page_scan_tab_contents

SBB SocIedade BotânICA Do Brasil. (10 de abril de 2012). Graziela Maciel Barroso: uma trajetória na ciência botânica do século XX. Recuperado de http://www.botanica.org.br/go_news.php?id=62

SEDEÑN, E. P. (2000). Institucionalización de la ciencia, valores epistémicos y contextuales: un caso ejemplar. Cadernos Pagu, (15), 77-102.

SHTEIR, A. B. (1997). Gender and "Modern" Botany in Victorian England. Osiris, 12, 29-38.

(1999). Cultivating women, cultivating science: Flora's daughters and botany in England, 1760 to 1860. Baltimore \& London: Johns Hopkins University Press. 
(2007). Flora primavera or Flora meretrix? Iconography, Gender, and Science. Studies in Eighteenth-Century Culture, 1(1), 147-168.

SILVA, E. B. (1998). Des-construindo gênero em ciência e tecnologia. Cadernos Pagu, (10), 7-20.

Sistema Regional de Información en Línea para Revistas Científicas de América Latlna, el Caribe, España y Portugal. (17 de febrero de 1997). Búsqueda avanzada. Recuperado de http://www.latindex.org/latindex/inicio

Smithsonian National Museum of Natural History. (2017). José Cuatrecasas Medal for excellence in Tropical Botany. Recuperado de http:// botany.si.edu/cuatrecasas/cuatrecasas Medal.cfm

Sociedad Argentina de Botánica. (2010-2016). Boletín de la Sociedad Argentina de Botánica. Córdoba, Argentina. Recuperado de http:// www.scielo.org.ar/scielo.php?script=sci_serial \&pid=1851-2372

Sociedad BoliviAna de BotánICA. (2010-2016). Sociedad Boliviana de Botánica. Recuperado de http://boliviabotanica.org/resbbo/

SociedAd BotánICA DE MÉxICO. (2010-2016). Botanical Sciences. Formerly Boletín de la Sociedad Botánica de México. México. Recuperado de http:// www.botanicalsciences. com.mx/

Sombrio, M. M. de O., Lopes, M. M. y Velho, L. M. L. S. (2008). Práticas e disputas em torno do patrimônio cientifico-cultural-Bertha Lutz no conselho de fiscalização das expedições artísticas e científicas do Brasil. Varia História, 39(24), 311-327.

Sugimoto, C. R., Larivière, V. N. C., Gingras, Y. y Cronin, B. (2013). Global gender disparities in science. Nature, 504(7479), 211-213.

Tosı, L. (1998). Mulher e ciência: a revolução científica, a caça às bruxas e a ciência moderna. Cadernos Pagu, (10), 369-397. 


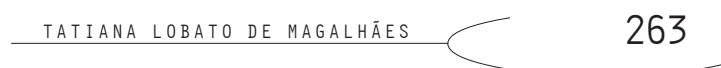

VEGA, R. (2014). Zoología y Botánica en los impresos femeninos de la Ciudad de México, 1839-1856. Iberoamericana, 13(51), 27-46.

VELHO, L. y LÉON, E. (1998). A construção social da produção científica por mulheres. Cadernos Pagu, (10), 309-344.

Vieira, A. O. S. y Gasper, A. L. (2015). Redes de Herbários e Herbários Virtuais no Brasil. Bioscience, 7(4), 3-23.

WollstoneCraft, M. (2009). A Vindication of the rights of woman: with strictures on political and moral subjects. Reino Unido: Oxford University Press.

Zamudio Ruiz, S. (2014). Luz María Villarreal de Puga (1913-2013). Acta Botánica Mexicana, (107), 1-3. 